\title{
Recurrent Staphylococcal Scalded Skin Syndrome in an Extremely Low-Birth-Weight Neonate
}

\author{
Jennifer Davidson, DO ${ }^{1}$ Samantha Polly, $\mathrm{BA}^{2} \quad$ Peter J. Hayes, $\mathrm{MD}^{3} \quad$ Kristopher R. Fisher, $\mathrm{MD}^{3}$ \\ Ajay J. Talati, MD ${ }^{1}$ Tejesh Patel, $M^{3}$ \\ ${ }^{1}$ Department of Pediatrics, University of Tennessee Health Science \\ Center, Memphis, Tennessee \\ ${ }^{2}$ College of Medicine, University of Tennessee Health Science Center, \\ College of Medicine, Memphis, Tennessee \\ ${ }^{3}$ Department of Dermatology, University of Tennessee Health \\ Science Center, Memphis, Tennessee \\ Address for correspondence Jennifer Davidson, DO, Neonatal- \\ Perinatal Fellow, University of Tennessee Health Science Center, \\ 853 Jefferson Ave., Suite 201, Memphis TN 38163 \\ (e-mail: jhendr20@uthsc.edu). \\ Am J Perinatol Rep 2017;7:e134-e137.
Abstract
Keywords
- staphylococcal scalded skin
- neonate
- low-birth-weight infant
- premature infants \\ syndrome \\ Staphylococcal scalded skin syndrome (SSSS) in premature infants is a rare condition. \\ We present SSSS in an extremely low-birth-weight (ELBW) infant with recurrent and \\ confirmed bacterial sepsis. We present it to emphasize the importance for clinicians to \\ not only recognize the clinical manifestations of SSSS, but also the need to closely \\ monitor infants, especially very low-birth-weight (VLBW) and ELBW infants with SSSS \\ for recurrence and bacterial sepsis. SSSS in preterm infants is a potentially lethal \\ condition and early recognition and appropriate supportive care could be life-saving.
}

Staphylococcal scalded skin syndrome (SSSS) is a blistering disease caused by exfoliative toxins (ETs) A and B. These toxins cleave the stratum granulosum layer of the epidermis leaving generalized blisters on all parts of the epidermis. ${ }^{1}$ Infants with an immature renal function have a difficult time clearing the toxins. ${ }^{2}$ SSSS has been documented in premature infants and very low-birth-weight infants (VLBW) including rare reports of recurrent disease. ${ }^{3-5}$ We describe a unique case of an extremely low-birth-weight (ELBW) infant who had SSSS followed by a recurrence of the disease with blood stream infection after a completion of appropriate antibiotic therapy.

\section{Case}

A $550 \mathrm{~g}$ female infant with an estimated 25 weeks of gestational age (GA) was delivered by emergent cesarean section due to preterm labor and breech presentation. Membranes were intact and amniotic fluid was clear at the time of delivery. Her mother was a 31-year-old, African-American, gravida 6 para 5 with only one prenatal visit to confirm pregnancy. All prenatal laboratory tests were within normal limits. The infant was intubated in the delivery room by the neonatal team and given surfactant. Apgar scores were 2, 3, and 5 at 1, 5, and 10 minutes, respectively.

The clinical course for the initial few weeks was typical for an ELBW preterm infant. She required mechanical ventilation with endotracheal tube in place until day of life (DOL) 26 at which respiratory support was lowered to noninvasive positive pressure ventilation. On DOL 27, she was reintubated for hypercarbia. Subsequently, within 24 hours, she developed signs of sepsis, including tachycardia, increased supplemental oxygen requirements, and emesis. Laboratory tests showed an elevated serum C-reactive protein (CRP) with normal white blood cells (WBCs). Intravenous antibiotics were initiated. Within 12 hours of onset of sepsis, skin received

February 21, 2017

accepted after revision

March 27, 2017
DOI https://doi.org/

10.1055/s-0037-1603971. ISSN 2157-6998.
Copyright $\odot 2017$ by Thieme Medical

Publishers, Inc., 333 Seventh Avenue, New York, NY 10001, USA. Tel: +1(212) 584-4662.
License terms

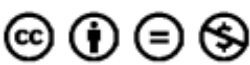




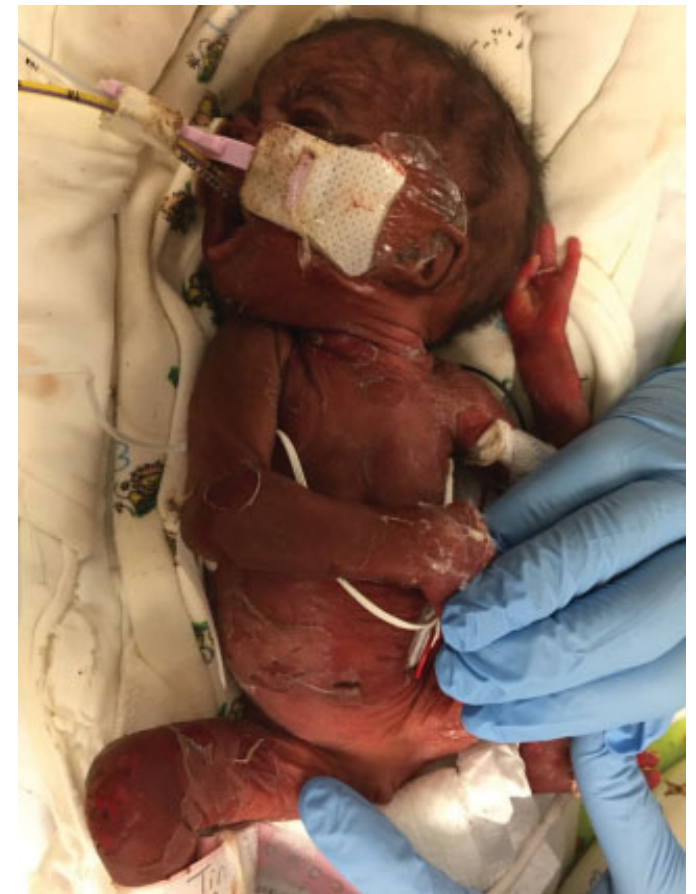

Fig. 1 Upper extremity erythroderma with diffuse superficial desquamation.

desquamation, especially on her face with areas of erythema and crusted erosions around her nasal area were noted. Within the subsequent few hours, she was noted to have diffuse erythrodema and superficial desquamation of her face, hands, arms, trunk, and legs (-Figs. 1-3). Clindamycin was initiated to help decrease staphylococcal toxin production. Blood cultures remained sterile but surface cultures from skin fluid, throat, and nares demonstrated methicillinsensitive Staphylococcus aureus (MSSA). To confirm the diagnosis, sheets of desquamating skin were removed and sent for histologic examination. The examination revealed an intraepidermal split at the level of the stratum granulosum, which is characteristic of SSSS (- Fig. $\mathbf{4 A}$ and $\mathbf{4 B}$ ). She

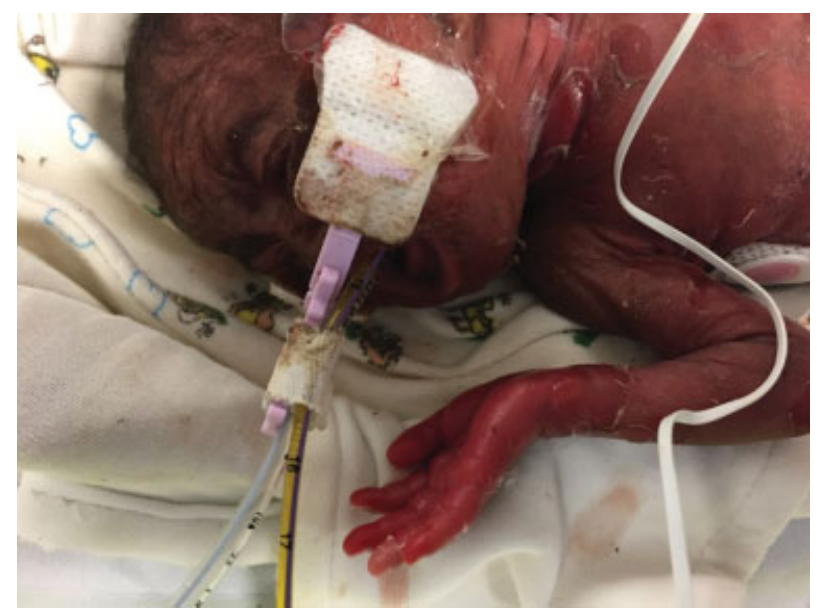

Fig. 2 Face and neck erythroderma with diffuse superficial desquamation.

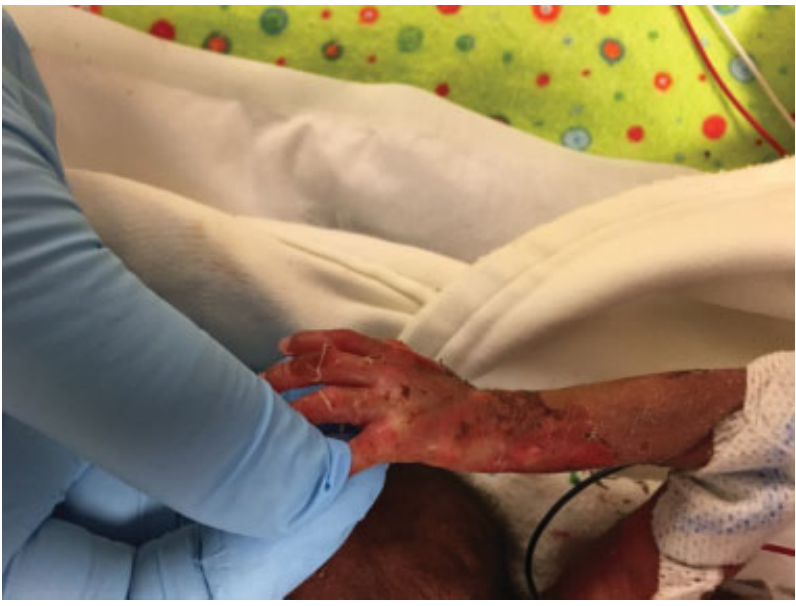

Fig. 3 Generalized erythroderma with diffuse superficial desquamation.

completed a 10-day course of nafcillin and clindamycin for appropriate coverage of MSSA. At the time of completion of antibiotic therapy, only one small area of superficial denuded skin over the extensor knee remained, which healed the following day. The remainder of her skin looked normal. The infant's general clinical condition improved and she was on baseline respiratory support.

About 10 days after discontinuing antibiotics, the infant's respiratory condition deteriorated and required endotracheal intubation for recurrent episodes of apnea. Within subsequent hours, she developed tachycardia, increased agitation, and skin desquamation on face, upper chest, right axilla, abdomen, back, and buttocks. Antibiotics were initiated again. Cultures revealed Enterobacter cloacae complex and Proteus mirabilis in addition to MSSA in the bloodstream. Cerebrospinal fluid (CSF) was obtained and did not reveal any microorganisms. In addition, there were no clinical signs of meningitis, such as lethargy, seizures, vomiting, or excess
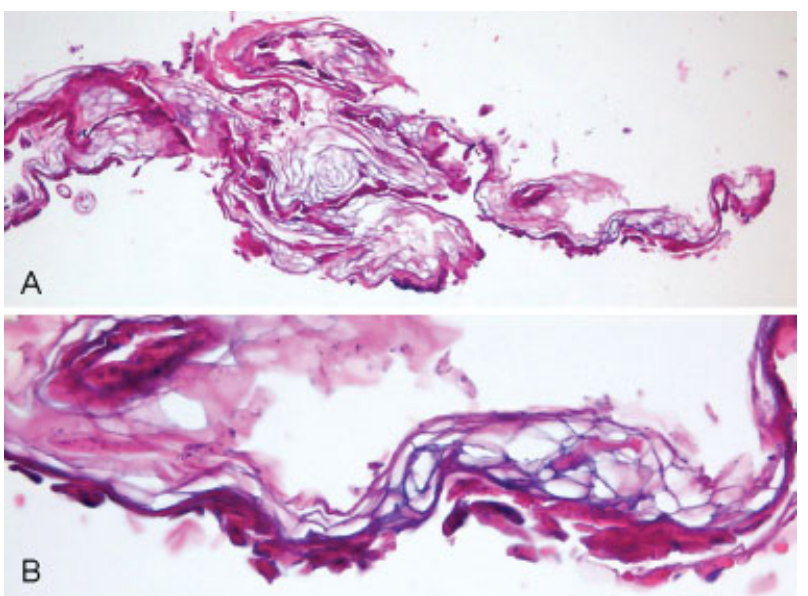

Fig. 4 Histopathology at low power (A) and high power (B) magnification revealed intraepidermal split at the level of the stratum granulosum, which is characteristic of staphylococcal scalded skin syndrome (SSSS). 
irritability. Repeat blood culture was negative. She completed a 10-day course of linezolid for recurrent SSSS and a 21-day course of meropenem for presumed Enterobacter meningitis. Skin lesions again resolved by the end of therapy, and respiratory status and general condition returned to baseline. The infant continued to improve during rest of neonatal intensive care unit (NICU) stay and was discharged home to family with no respiratory support, thermal stability, and full-volume enteral feeding on DOL 109 and corrected GA of 40 weeks.

\section{Discussion}

SSSS, also referred to as pemphigus neonatorum, is an exfoliative dermatitis caused by toxin-producing strains of S. aureus. Specifically, these phage-infected bacteria produce exfoliative toxins A and B (ETA and ETB) that target desmoglein 1 , a cadherin molecule that mediates keratinocyte cell adhesion in the stratum granulosum layer of the epidermis. ${ }^{6}$ While the dermatologic manifestations of infection with $S$. aureus can range from localized impetigo to widespread bullae and erythrodermic exfoliation, only $5 \%$ of strains produce the exfoliative toxins responsible for SSSS. ${ }^{7}$

Clinically, SSSS is most commonly seen in children between the age of 6 months and 5 years with the likelihood of disease highest between 2 and 3 years of age. ${ }^{8}$ Normally, SSSS is a clinical diagnosis. Cultures should not be taken from intact or denuded bullae, as they are likely to be negative due to toxinproducing nature of the disease. Instead, cultures should be taken from mucosal surfaces, such as the nares, pharynx, or conjunctiva, to confirm the presence of $S$. aureus. In neonates with a diffuse desquamating and blistering rash, viral or druginduced Stevens-Johnson syndrome/toxic epidermal necrolysis and epidermolysis bullosa also remain high on the differential diagnosis. In these instances, skin biopsy for histologic examination is extremely helpful. ${ }^{9}$ Biopsies of SSSS will reveal a split at the level of stratum granulosum in the epidermis. Adequate tissue sampling for diagnosis can be obtained from sheets of desquamating skin. ${ }^{10}$ According to the 30th edition of Red Book, ${ }^{1}$ any infant with a concern for SSSS who is moderately ill with an extensive area of skin involvement or is critically ill should be started on vancomycin until susceptibilities are identified. In a critically ill infant, nafcillin is also recommended. ${ }^{1}$ Clindamycin is a suppressor of the bacterial toxin production. Since SSSS is a toxin-mediated illness, clindamycin may be beneficial. However, evidence behind its routine use in SSSS is lacking. ${ }^{11}$ The 2011 Infectious Diseases Society of America (IDSA) MRSA guidelines also do not recommend routine use of clindamycin or linezolid as adjunctive therapies for invasive MRSA infections, but state that some experts may consider clindamycin or linezolid in selected scenarios. $^{12}$

Despite effective and known treatment, mortality in children still ranges between 3 and $11 \%$ and is usually secondary to a disease complication, such as dehydration or superinfection. ${ }^{13}$ Interestingly, Kapoor et al noted that the unique distribution of desmoglein 1 and 3 in neonatal epidermis should protect the neonates against SSSS. Unlike adults, in whom desmoglein 3 is present only in deeper layers of skin, in neonates, it is found alongside desmoglein 1 in the epidermis. Therefore, it remains to be elucidated how ETA and ETB are able to cause blistering and desquamation by targeting desmoglein 1 alone with desmoglein 3 in place to provide keratinocyte adhesion. ${ }^{14}$ In reality, however, though rare, SSSS is reported in neonates with an onset typically between the third and 16th day of life, and it rarely develops in premature and very low-birth-weight infants. ${ }^{15}$

When SSSS does occur in neonates, it has rarely been reported to recur and seldom is associated with bacterial septicemia. We describe a case of SSSS in an ELBW infant with recurrent and confirmed bacterial sepsis; to our knowledge, this is the first case presented with both of these findings.

Recurrent SSSS in neonates is extremely rare. A literature review revealed only 3 cases. $^{3-5}$ Both Duijsters et al and Rieger-Fackeldey et al reported cases of premature infants with recurrent SSSS that occurred less than 1 month after initial diagnosis and treatment. In each case, the neonates developed no further complications after a second course of antibiotics and neither reported positive blood cultures during infection., ${ }^{3,4}$ An additional case report from 2005 described two Taiwanese siblings with recurrent SSSS secondary to Netherton syndrome that causes defective regulation of the skin barrier and immunity. ${ }^{5}$

Reports of neonatal staphylococcal septicemia occurring in conjunction with a diagnosis of SSSS are equally as rare as recurrence of SSSS. Only four cases in the literature are reported. Each of these cases affected premature infants, with two occurring more than 23 days after birth and the remaining two during the first week of life. Hutten et al described a case in which the affected neonate actually presented with symptoms of sepsis and only began to manifest the dermatologic signs of SSSS during his admission. ${ }^{16}$ Another case was also complicated by a concomitant varicella infection. ${ }^{17}$ Two other case studies reported fatal septicemia due to Pseudomonas aeruginosa following development of SSSS in neonates. ${ }^{18,19}$

The recurrence in our infant may be secondary to the combination of decreased renal clearance of exotoxins in addition to the immature immune system of ELBW infant and inability to develop antibody response to the toxin. Renal immaturity in infants decreases the ability to clear the epidermotrophic toxins. In mice models, a critical toxin level is required for a sustained time period to cause exfoliation. ${ }^{20}$ Furthermore, the levels of antitoxin antibodies are agedependent. At birth in term infants, anti-ETA antibodies are detected in $88 \%$ of cord blood and this level decreases to $30 \%$ by 3 months. Saida et al found that the prevalence of anti-ETA antibodies were significantly lower in preterm $<30$ weeks rather than term infants, which may place these neonates at an increased risk of SSSS. ${ }^{8}$ They further hypothesized that decreased anti-toxin immunoglobulin $(\operatorname{Ig} G)$ levels cause the disease and that other factors such as immunologic immaturity affect severity.

As with any infection, prevention is a high priority. S. aureus is usually hospital acquired in premature infants. 
Personal barrier techniques are the most effective way to prevent transmission of infection. Proper hand washing, minimal handling, appropriately cleaning equipment between uses, and avoiding central catheterization are also methods to prevent spread of infection. ${ }^{1,21}$ There are only a few reported cases of recurrent SSSS in the literature; therefore, we are left with very little guidance on how to prevent relapse. The hygiene measures mentioned above are just as important in primary prevention as they are in prevention of relapse. Currently, there is no evidence to support treating these infants with a longer course of antibiotics or using a different type of antibiotic to prevent recurrence. After clearance of bacteremia, persistent colonization of skin and mucosal surfaces may be difficult to treat; so, repeat skin and mucosal cultures may be of little benefit. To our knowledge, exfoliative toxin measurements have only been utilized in research studies and may not be available as a standard test. ${ }^{22,23}$

In conclusion, we believe this to be the first case of SSSS in an ELBW infant with recurrent and confirmed bacterial sepsis. We present it to emphasize the importance for clinicians to not only recognize the clinical manifestations of SSSS, but also the need to closely monitor infants, especially VLBW and ELBW infants with SSSS for recurrent and bacterial sepsis. Though our infant survived to discharge and is doing well at 1-year of age without reoccurrence, SSSS in preterm infants is still a potentially lethal condition and early recognition and appropriate supportive care could be lifesaving.

\section{References}

1 Red Book, 30th Edition. 2015. Report on the Committee on Infectious Diseases, 30th Edition. In: Kimberlin DW, Brady MT, Jackson MA, Long SS eds. Elk Grove Village, IL: American Academy of Pediatric; 2015

2 Pollack S. Staphylococcal scalded skin syndrome. Pediatrics in review/American. Acad Pediatr 1996;17(01):18

3 Duijsters CE, Halbertsma FJ, Kornelisse RF, Arents NL, Andriessen P. Recurring staphylococcal scalded skin syndrome in a very low birth weight infant: a case report. J Med Case Reports 2009;3(01): 7313

4 Rieger-Fackeldey E, Plano LR, Kramer A, Schulze A. Staphylococcal scalded skin syndrome related to an exfoliative toxin A- and B-producing strain in preterm infants. . Eur J Pediatr 2002;161 (12):649-652

5 Chao SC, Richard G, Lee JY. Netherton syndrome: report of two Taiwanese siblings with staphylococcal scalded skin syndrome and mutation of SPINK5. Br J Dermatol 2005;152(01):159-165

6 Amagai M, Yamaguchi T, Hanakawa Y, Nishifuji K, Sugai M, Stanley JR. Staphylococcal exfoliative toxin B specifically cleaves desmoglein 1. J Invest Dermatol 2002;118(05):845-850
7 Elsner P, Hartmann A. Epidemiology of ETA-Producing and ETBProducing Staphylococci in Dermatological Patients. Paper presented at: Zentralblatt Fur Bakteriologie Mikrobiologie Und Hygiene Series A-Medical Microbiology Infectious Disease Virology Parasitology 1988

8 Saida K, Kawasaki K, Hirabayashi K, et al. Exfoliative toxin A staphylococcal scalded skin syndrome in preterm infants. Eur J Pediatr 2015;174(04):551-555

9 Ladhani S, Joannou CL. Difficulties in diagnosis and management of the staphylococcal scalded skin syndrome. Pediatr Infect Dis J 2000;19(09):819-821

10 Elston DM, Stratman EJ, Miller SJ. Skin biopsy: biopsy issues in specific diseases. J Am Acad Dermatol 2016;74(01):1-16; quiz $17-18$

11 Long SS, Pickering LK, Prober CG. Principles and practice of pediatric infectious disease. Elsevier Health Sciences; 2012

12 Liu C, Bayer A, Cosgrove SE, et al. Clinical practice guidelines by the Infectious Diseases Society of America for the treatment of methicillin-resistant Staphylococcus aureus infections in adults and children. Clin Infect Dis 2011;52(03):e18-e55. doi: 10.1093/ cid/ciq146

13 Mockenhaupt M, Idzko M, Grosber M, Schöpf E, Norgauer J. Epidemiology of staphylococcal scalded skin syndrome in Germany. J Invest Dermatol 2005;124(04):700-703

14 Kapoor V, Travadi J, Braye S. Staphylococcal scalded skin syndrome in an extremely premature neonate: a case report with a brief review of literature. J Paediatr Child Health 2008;44(06): 374-376

15 Dancer SJ, Simmons NA, Poston SM, Noble WC. Outbreak of staphylococcal scalded skin syndrome among neonates. J Infect 1988;16(01):87-103

16 Hütten M, Heimann K, Baron JM, Wenzl TG, Merk HF, Ott H. Staphylococcal scalded skin syndrome as a harbinger of lateonset staphylococcal septicaemia in a premature infant of very low birth weight. Acta Derm Venereol 2008;88(04):416-417

17 Singh SN, Tahazzul M, Singh A, Chandra S. Varicella infection in a neonate with subsequent staphylococcal scalded skin syndrome and fatal shock. BMJ Case Rep 2012;2012. pii:bcr2012006462

18 Hörner A, Hörner R, Salla A, et al. Staphylococcal scalded skin syndrome in a premature newborn caused by methicillin-resistant Staphylococcus aureus: case report. Sao Paulo Med J 2015; 133(05):450-453

19 Hoffmann R, Lohner M, Böhm N, Schaefer HE, Leititis J. Staphylococcal scalded skin syndrome (SSSS) and consecutive septicaemia in a preterm infant. Pathol Res Pract 1994;190(01):77-81; discussion 81-83

20 Cribier B, Piemont Y, Grosshans E. Staphylococcal scalded skin syndrome in adults. A clinical review illustrated with a new case. J Am Acad Dermatol 1994;30(2, Pt 2):319-324

21 Ladhani S, Joannou CL, Lochrie DP, Evans RW, Poston SM. Clinical, microbial, and biochemical aspects of the exfoliative toxins causing staphylococcal scalded-skin syndrome. Clin Microbiol Rev 1999;12(02):224-242

22 Saiman L, Jakob K, Holmes KW, et al. Molecular epidemiology of staphylococcal scalded skin syndrome in premature infants. Pediatr Infect Dis J 1998;17(04):329-334

23 Ladhani S. Recent developments in staphylococcal scalded skin syndrome. Clin Microbiol Infect 2001;7(06):301-307 